\title{
Reintroduction of immunosuppressive medications in pediatric rheumatology patients with histoplasmosis: a case series
}

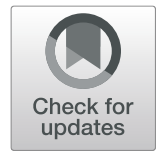

\author{
Rachel A. Brown ${ }^{1}$, Fatima Barbar-Smiley², Cagri Yildirim-Toruner², Monica I. Ardura², Stacy P. Ardoin² and
} Shoghik Akoghlanian $2,3^{*}$

\begin{abstract}
Background: Children with rheumatic diseases (CRD) receiving immunosuppressive medications (IM) are at a higher risk for acquiring potentially lethal pathogens, including Histoplasma capsulatum (histoplasmosis), a fungal infection that can lead to prolonged hospitalization, organ damage, and death. Withholding IM during serious infections is recommended yet poses risk of rheumatic disease flares. Conversely, reinitiating IM increases risk for infection recurrence. Tumor necrosis factor alpha inhibitor (TNFai) biologic therapy carries the highest risk for histoplasmosis infection after epidemiological exposure, so other IM are preferred during active histoplasmosis infection. There is limited guidance as to when and how IM can be reinitiated in CRD with histoplasmosis. This case series chronicles resumption of IM, including non-TNFai biologics, disease-modifying anti-rheumatic drugs (DMARDs), and corticosteroids, following histoplasmosis among CRD.
\end{abstract}

Case presentation: We examine clinical characteristics and outcomes of 9 patients with disseminated or pulmonary histoplasmosis and underlying rheumatic disease [juvenile idiopathic arthritis (JIA), childhood-onset systemic lupus erythematosus (CSLE), and mixed connective tissue disease (MCTD)] after reintroduction of IM. All DMARDs and biologics were halted at histoplasmosis diagnosis, except hydroxychloroquine (HCQ), and patients began antifungals. Following IM discontinuation, all patients required systemic or intra-articular steroids during histoplasmosis treatment, with 4/9 showing Cushingoid features. Four patients began new IM regimens [2 abatacept (ABA), $1 \mathrm{HCQ}$, and 1 methotrexate (MTX)] while still positive for histoplasmosis, with 3/4 (ABA, MTX, HCQ) later clearing their histoplasmosis and 1 (ABA) showing decreasing antigenemia. Collectively, 8/9 patients initiated or continued DMARDs and/or non-TNFai biologic use (5 ABA, 1 tocilizumab, 1 ustekinumab, 3 MTX, 4 HCQ, 1 leflunomide). No fatalities, exacerbations, or recurrences of histoplasmosis occurred during follow-up (median 33 months).

\footnotetext{
* Correspondence: shoghik.akoghlanian@nationwidechildrens.org

${ }^{2}$ Nationwide Children's Hospital, Ohio, Columbus, USA

${ }^{3}$ Department of Pediatric Rheumatology, Nationwide Children's Hospital, 555

S. 18th St, OH 43205 Columbus, USA

Full list of author information is available at the end of the article
}

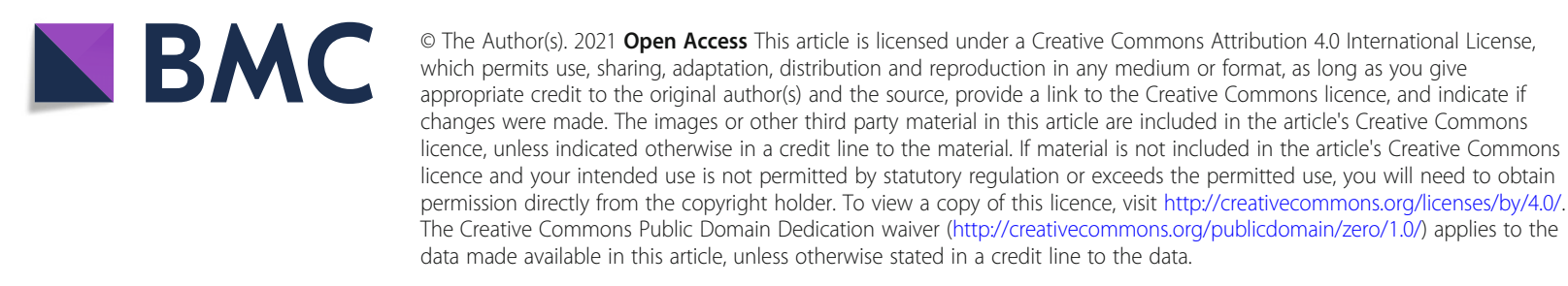




\begin{abstract}
Conclusions: In our cohort of CRD, histoplasmosis course following reintroduction of non-TNFai IM was favorable, but additional studies are needed to evaluate optimal IM management during acute histoplasmosis and recovery. In this case series, non-TNFai biologic, DMARD, and steroid treatments did not appear to cause histoplasmosis recurrence. Adverse events from corticosteroid use were common. Further research is needed to implement guidelines for optimal use of non-TNFai (like ABA), DMARDs, and corticosteroids in cRD following histoplasmosis presentation.
\end{abstract}

Keywords: histoplasmosis, juvenile idiopathic arthritis, immunosuppression, tumor necrosis factor alpha inhibitor, corticosteroid, abatacept, DMARD, Cushing's syndrome

\section{Background}

Over 300,000 children in the USA live with juvenile idiopathic arthritis (JIA) and other chronic rheumatic diseases. Advances in understanding the cytokine-driven pathogenesis of rheumatic diseases have provided potent therapeutics. A growing number of FDA approved biologics targeting cytokines are available, including inhibitors of the pro-inflammatory mediators tumor necrosis factor alpha (TNFai), interleukin (IL)-1b (canakinumab), IL-1R (anakinra), IL-6R (tocilizumab), as well as T-cell co-stimulation (abatacept $[\mathrm{ABA}]$ ) and B-cells (rituximab) [1]. However, cytokines are also critical to control and clear infections, and autoimmunity itself increases infection risk due to immune system dysregulation [1]. Immunosuppressive medications (IM) heighten risk of developing serious infections, including granulomatous infections like histoplasmosis [1-3]. Histoplasmosis is caused by inhaling spores of Histoplasma capsulatum, a fungus found in soil contaminated with bird or bat droppings and endemic to areas such as the Ohio and Mississippi River Valleys [4-6]. While typically causing mild or asymptomatic pulmonary infection in immunocompetent patients, $H$. capsulatum can disseminate systemically in susceptible hosts, leading to poor outcomes and requiring a prolonged antifungal course [6-8]. Furthermore, itraconazole, the recommended oral antifungal for H. capsulatum, can result in drug interactions due to inhibiting the CYP3A4 pathway [9-13].

Our center at Nationwide Children's Hospital $(\mathrm{NCH})$ in Columbus, Ohio facilitates over 6,000 visits per year for children with rheumatic diseases (cRD). Standard rheumatic treatments include nonsteroidal antiinflammatory drugs (NSAIDs), corticosteroids, and disease-modifying anti-rheumatic drugs (DMARDs) (methotrexate [MTX], leflunomide, hydroxychloroquine [HCQ], etc.), but many of these patients are or were also managed with TNFa antagonists (etanercept, infliximab, adalimumab, etc.) or other biologics at some point. It is controversial whether low dose MTX increases infection susceptibility, whereas combination IM therapy, corticosteroids, or biologics do, while HCQ does not $[1,3,14$, $16,17]$. Indeed, TNFai complicated by infections is well reported $[3,8,17-22]$. For patients receiving TNFai who present with fever or pulmonary symptoms, extensive infectious workup is undertaken given the broad infectious differential. It is recommended that IM be held during acute infections, but this interruption in treatment may lead to a rheumatic disease flare. Optimal rheumatic disease management is unknown, including the duration of IM discontinuation and, importantly, safety data about reintroducing TNFai or other biologics are largely lacking $[1,3,23,24,22]$. Meta-analyses suggest some biologics carry a lower infection risk compared to TNFai [25]. For example, an observational study of adult patients with rheumatoid arthritis (RA) noted participants receiving $\mathrm{ABA}$ had lower rates of infection-related hospitalization compared with other biologics [26]. However, utilization of these non-TNFai following histoplasmosis diagnosis (FHD) and histoplasmosis clearance (HC) has not been reported and merits further scrutiny. In our report, we describe the treatment course, IM management, and outcomes of cRD diagnosed with histoplasmosis.

\section{Methods}

A retrospective chart review of electronic health records identified children followed for rheumatic disease management at $\mathrm{NCH}$, who were diagnosed with and treated for proven, probable, or possible histoplasmosis between January 2011 and January 2018. Primary inclusion criteria included: diagnosis with a pediatric rheumatic disease and a later/concurrent diagnosis of histoplasmosis. Data were collected for IMs prior to and following histoplasmosis diagnosis and histoplasmosis course. IMs were characterized as (1) biologic DMARDs, such as TNFai or non-TNFai, (2) non-biologic DMARDs, and (3) corticosteroids, including intra-articular steroid injections (IASIs) and oral/inhaled/intermuscular/intravenous (systemic) steroids. Systemic steroids were classified as "burst" (high dose $>10 \mathrm{mg} /$ day provided to treat active rheumatic or non-rheumatic disease, like asthma) or "maintenance" (low dose $\leq 10 \mathrm{mg} /$ day). Histoplasmosis resolution was defined as clearance of urine and serum antigens and clinical resolution of signs and symptoms. This study was reviewed and approved by the local Institutional Review Board IRB17-01241. 


\section{Results}

Nine cRD patients (78\% female) met inclusion criteria, including $7(78 \%)$ patients with JIA (2 psoriatic, 2 polyarticular, 2 enthesitis-related arthritis [ERA], and 1 oligoarticular), 1 childhood-onset systemic lupus erythematosus (cSLE), and 1 mixed connective tissue disease (MCTD) patient. Patient demographics and prehistoplasmosis diagnosis medications are shown in Table 1. Five out of 7 JIA patients were receiving IM prior to infection diagnosis: 1 was receiving MTX alone and 4 were receiving combination therapy with MTX and TNFai ( 2 adalimumab, 1 etanercept, 1 infliximab), including one patient also receiving burst steroids. The other 2 patients with JIA were managed with NSAIDs. Both CSLE and MCTD patients were on MTX, HCQ, and systemic steroids (burst and maintenance, respectively). Median time from starting TNFai to histoplasmosis manifestation was 1.3 months (range 0.5-14.1) compared with 18 months (4.6-32) and 13 months (0.531) for DMARDs and steroids, respectively.

Histoplasmosis diagnosis and course are shown in Table 2 . Three (33\%) patients were able to identify potential exposures (recent bonfire, farm residence, and birds/bats in attic). H. capsulatum was detected by bronchoalveolar lavage, pulmonary wedge biopsy, Histoplasma antigen, and/or serology for a total of 3 proven, 5 probable, and 1 possible histoplasmosis cases [27]. Five (56\%) patients developed disseminated histoplasmosis (DH), and 3 (33\%) developed pulmonary histoplasmosis (PH). The possible case was not further categorized as $\mathrm{PH}$ or $\mathrm{DH}$ but included in this series due to positive serology and clinical symptoms (data not shown). For the 5
DH patients, 2 were receiving both TNFai and MTX, 1 was receiving TNFai, MTX, and steroid bursts, 1 was receiving DMARDs only, and 1 was receiving DMARD and maintenance steroids. Of the $3 \mathrm{PH}$ patients, 1 was receiving TNFai and MTX and 2 were not receiving IMs. The patient with the possible histoplasmosis infection was receiving DMARDs and burst steroids. At histoplasmosis diagnosis, IM, except HCQ and steroids, were held. Six (67\%) patients (5 DH and $1 \mathrm{PH}$ ) required hospitalization for acute histoplasmosis for a median of 14 days [6-22], with $1 \mathrm{DH}$ patient needing admission to the intensive care unit for mechanical ventilation for 14 days. During follow up, $7 / 9$ (78\%) demonstrated complete resolution of antigenemia and clinical symptoms with a median time to clearance of 12.7 months (2.3-37.2) FHD and 2 were clinically asymptomatic at 11 and 25 months FHD while showing low antigenemia. Four of nine (44\%) patients continued prophylactic itraconazole after $\mathrm{HC}$ for a median of 23.6 months (5.0-70.0). Median follow-up after HC was 29.3 months (0-70.3), with a total follow-up from histoplasmosis diagnosis of 33 months (11-88.5). There was no observed mortality or histoplasmosis relapse during this time.

\section{Patient Use of Immunosuppressive Medications Following Histoplasmosis Infection was Necessary}

Patients initially managed their underlying rheumatic conditions during antifungal therapy with NSAIDs or $\mathrm{HCQ}$, but while receiving these medications all patients experienced rheumatic disease flare requiring further intervention.

Table 1 Characteristics of patients at time of histoplasmosis diagnosis. Information was collected on patient demographics and IM prior to histoplasmosis diagnosis

\begin{tabular}{|c|c|c|c|c|}
\hline Patient & $\begin{array}{l}\text { Age (yr) at } \\
\text { Histoplasmosis } \\
\text { Diagnosis, Sex }\end{array}$ & $\begin{array}{l}\text { Rheumatic } \\
\text { Disease }\end{array}$ & $\begin{array}{l}\text { Anti-TNFa (duration of Therapy in Months } \\
\text { Prior to Histoplasmosis Diagnosis) }\end{array}$ & $\begin{array}{l}\text { Other Pre-histoplasmosis IM } \\
\text { (month duration) }\end{array}$ \\
\hline 1 & $11, F$ & JA, Psoriatic & Adalimumab (0.5) & MTX (5) \\
\hline 2 & $17, F$ & JIA, Poly & Etanercept (1.3) & MTX (7), burst steroids (1) \\
\hline 3 & $18, F$ & JA, Psoriatic & Infliximab (14.1) & MTX (23) \\
\hline 4 & $15, F$ & cSLE & - & $\begin{array}{l}\text { MTX (3), HCQ (5), burst } \\
\text { steroids (12.8) }\end{array}$ \\
\hline 5 & $16, F$ & JIA, Enthesitis & - & None \\
\hline 6 & $9, M$ & JIA, Enthesitis & Adalimumab (1.3) & MTX (15) \\
\hline 7 & $17, F$ & JIA, Oligo & - & MTX (18) \\
\hline 8 & $16, M$ & MCTD & - & $\begin{array}{l}\text { MTX (31), HCQ (32), } \\
\text { maintenance steroids (30) }\end{array}$ \\
\hline 9 & $5, F$ & JIA, Poly & - & None \\
\hline $\begin{array}{l}\text { Summary, \% or } \\
\text { Median (range): }\end{array}$ & $\begin{array}{l}16[5-18] \\
78 \% \mathrm{~F}\end{array}$ & $\begin{array}{l}7 \text { JIA, } 1 \text { CSLE, } \\
1 \text { MCTD }\end{array}$ & 44 \% TNFai, 1.3 (0.5-14.1) & $\begin{array}{l}78 \% \text { DMARD, } 18(4.6-32) \\
33 \% \text { Steroids, } 13(0.6-30)\end{array}$ \\
\hline
\end{tabular}

Key: $\mathrm{Pt}=$ patient identifier; $\mathrm{yr}=$ years; IM = immunosuppressive medication; JIA = juvenile idiopathic arthritis, MTX = methotrexate, Poly = polyarticular; $\mathrm{CSLE}=$ childhood-onset systemic lupus erythematosus; $\mathrm{HCQ}=$ hydroxychloroquine; Oligo = oligoarticular; $\mathrm{MCTD}=$ mixed connective tissue disease 
Table 2 Histoplasmosis presentation. Data were collected for histoplasmosis infection, type, hospitalization, diagnostic tests, treatment, and outcomes

\begin{tabular}{|c|c|c|c|c|c|c|c|c|c|}
\hline $\mathrm{Pt}$ & $\begin{array}{l}\text { Known } \\
\text { Exposure }\end{array}$ & $\begin{array}{l}\text { Histoplasmosis } \\
\text { Type }\end{array}$ & $\begin{array}{l}\text { Histology } \\
\text { Staining }\end{array}$ & $\begin{array}{l}\text { Antigen } \\
\text { (Serum, } \\
\text { Urine) }\end{array}$ & Category & $\begin{array}{l}\text { Days } \\
\text { Hospitalized } \\
\text { (Intensive } \\
\text { care) }\end{array}$ & $\begin{array}{l}\text { Antifungal } \\
\text { Therapy }\end{array}$ & $\begin{array}{l}\text { Outcome (months } \\
\text { to HC or still } \\
\text { antigen }+ \text { ) } \\
\text { Itraconazole } \\
\text { prophylaxis } \\
\text { (months) }\end{array}$ & $\begin{array}{l}\text { Months Follow } \\
\text { Up After } \\
\text { Histoplasmosis } \\
\text { Diagnosis, } \\
\text { Clearance }\end{array}$ \\
\hline $1 \mathrm{JA}$ & No & $\mathrm{PH}$ & ND &,++ & Probable & 18 & $\begin{array}{l}\text { Amphotericin } \\
\text { B } \\
\text { Itraconazole }\end{array}$ & $\begin{array}{l}\text { HC at } 36.7 \\
\text { Prophylaxis for } 19.6\end{array}$ & $80,43.3$ \\
\hline $2 \mathrm{JIA}$ & No & $\mathrm{DH}$ & $+B A L$ &,++ & Proven & 11 & $\begin{array}{l}\text { Amphotericin } \\
\text { B } \\
\text { Itraconazole }\end{array}$ & $\begin{array}{l}\text { HC at } 37.2 \\
\text { Prophylaxis for } 5.0\end{array}$ & $89,51.8$ \\
\hline $3 \mathrm{JA}$ & No & $\mathrm{DH}$ & $\begin{array}{l}+ \\
\text { pulmonary } \\
\text { wedge } \\
\text { biopsy }\end{array}$ &,++ & Proven & $\begin{array}{l}22,(14, \\
\text { ventilation })\end{array}$ & $\begin{array}{l}\text { Amphotericin } \\
\text { B } \\
\text { Itraconazole }\end{array}$ & $\begin{array}{l}\mathrm{HC} \text { at } 12.7 \\
\text { Prophylaxis for } 70.0\end{array}$ & $83,70.3$ \\
\hline 4 CSLE & No & Uncategorized & ND &,-- & Possible & No & Itraconazole* & $\mathrm{HC}$ at 3.7 & $33,29.3$ \\
\hline $5 \mathrm{JA}$ & No & $\mathrm{PH}$ & ND &,-- & Probable & No & $\begin{array}{l}\text { Itraconazole } \\
\text { Fluconazole }\end{array}$ & $\mathrm{HC}$ at 2.3 & $28,25.7$ \\
\hline $6 \mathrm{JIA}$ & $\begin{array}{l}\text { Lives on } \\
\text { farm }\end{array}$ & $\mathrm{DH}$ & ND &,++ & Probable & 17 & $\begin{array}{l}\text { Amphotericin } \\
\text { B } \\
\text { Itraconazole }\end{array}$ & $\begin{array}{l}\mathrm{HC} \text { at } 21.2 \\
\text { Prophylaxis for } 27.5\end{array}$ & $67,45.8$ \\
\hline $7 \mathrm{JA}$ & $\begin{array}{l}\text { Attended } \\
\text { Bonfire }\end{array}$ & $\mathrm{DH}$ & ND &,++ & Probable & 6 & Itraconazole & $\begin{array}{l}\text { Asymptomatic } \\
\text { Antigen < LOQ at } \\
18.0+ \\
\text { Halted itraconazole } \\
18.0 \mathrm{FHD}\end{array}$ & $26, N A$ \\
\hline 8 MCTD & No & $\mathrm{DH}$ & ND &,++ & Probable & $\begin{array}{l}8\left(1^{* *}, \text { No }\right. \\
\text { ventilation) }\end{array}$ & $\begin{array}{l}\text { Amphotericin } \\
\text { B } \\
\text { Itraconazole }\end{array}$ & $\begin{array}{l}\text { Asymptomatic } \\
\text { Antigen decreasing } \\
\text { at } 11.0\end{array}$ & $11, \mathrm{NA}$ \\
\hline $9 \mathrm{JlA}$ & $\begin{array}{l}\text { Birds and } \\
\text { Bats in } \\
\text { attic }\end{array}$ & $\mathrm{PH}$ & $\begin{array}{l}+ \\
\text { pulmonary } \\
\text { wedge } \\
\text { biopsy }\end{array}$ & -- & Proven & No & Itraconazole & $\mathrm{HC}$ at 3.3 & $18,14.7$ \\
\hline $\begin{array}{l}\text { Summary: } \\
\text { Median } \\
\text { (range) }\end{array}$ & $\begin{array}{l}33 \% \text { Yes } \\
66 \% \text { No }\end{array}$ & $\begin{array}{l}56 \% \text { DH } \\
33 \% \text { PD } \\
11 \% \text { unnoted }\end{array}$ & $\begin{array}{l}3 \text { Positive } \\
6 \mathrm{ND}\end{array}$ & $\begin{array}{l}67 \% \\
\text { Antigen } \\
+(100 \% \\
\text { DH, } 33 \% \\
\text { PH) }\end{array}$ & $\begin{array}{l}3 \text { Proven } \\
5 \\
\text { Probable } \\
1 \\
\text { Possible }\end{array}$ & $\begin{array}{l}67 \% \text { for } 14 \\
(6-22) \\
11 \% \text { needing } \\
\text { ventilation }\end{array}$ & $\begin{array}{l}{ }^{*} 89 \% \\
\text { Itraconazole } \\
56 \% \\
\text { Amphotericin } \\
\text { B } \\
11 \% \\
\text { Fluconazole }\end{array}$ & $\begin{array}{l}78 \% \text { now HC } \\
12.7 \text { to } \mathrm{HC}(2.3-37.2) \\
44 \% \text { prophylaxis for } \\
23.6(5-70)\end{array}$ & $\begin{array}{l}\text { FHD } 33(11-88.5) \\
\text { HC } 29.3(0-70.3)\end{array}$ \\
\hline
\end{tabular}

*Given itraconazole, but declined to take it.**Intensive care stay during hospitalization was attributed to "possible shock 2nd to adrenal insufficiency." Key: $\mathrm{Pt}=$ Patient identifier and rheumatic disease; $\mathrm{PH}=$ Pulmonary histoplasmosis; $\mathrm{DH}=$ Disseminated histoplasmosis; $\mathrm{ND}=\mathrm{No}$ data; $+=\mathrm{Positive} ;-=\mathrm{Negative}$; $\mathrm{HC}=$ Histoplasmosis clearance; $\mathrm{BAL}=$ Bronchoalveolar lavage; $\mathrm{LOQ}=$ Limit of quantification; FHD = Following histoplasmosis diagnosis

\section{Steroids}

Prior to $\mathrm{HC}$ and while still taking itraconazole, all patients received steroidal therapy (Table 3), with 5/9 (56\%) receiving systemic steroids (including for nonrheumatic conditions or maintenance doses) and 8/9 (89\%) patients (7/7 JIA and 1 MCTD) receiving IASIs. Collectively, 4 received IASI and systemic steroids (including 1 continuing maintenance dosing), 4 received solely IASI, and 1 continued prior systemic steroid burst usage. Adverse events were common. One patient reported known corticosteroid allergies and developed sweating and chills limited to $24 \mathrm{~h}$. Another 4 patients developed days to months of typical Cushingoid features shortly after IASIs, including both $(2 / 2)$ patients receiving burst steroids and IASI as well as half $(2 / 4)$ of the patients just receiving IASI without any systemic steroids. The median time FHD was 4.3 months $(0-15.0)$ to starting systemic steroids (includes maintenance dosing) and 8.3 months (0.8-18.3) for IASI (Table 3$)$.

\section{Biologics and DMARDs}

Active rheumatic disease led to initiating biologics and DMARDs in 7 patients (Table 4) including: 3 ABA (with 
Table 3 Use of intra-articular steroid injections (IASI) and systemic steroids and adverse events during histoplasmosis treatment. Data for number and timing of IASI and systemic steroids were collected as well as concomitant use of itraconazole. Adverse events were noted

\begin{tabular}{|c|c|c|c|c|}
\hline Pt & $\begin{array}{l}\text { IASI (months after } \\
\text { histoplasmosis } \\
\text { diagnosis) }\end{array}$ & $\begin{array}{l}\text { Systemic Steroids (months } \\
\text { after histoplasmosis } \\
\text { diagnosis) }\end{array}$ & $\begin{array}{l}\text { Concomitant } \\
\text { Itraconazole? }\end{array}$ & Description of Adverse Event \\
\hline $1 \mathrm{JIA}$ & $3(7.2)$ & $\begin{array}{l}\text { Intermittent high/burst ( } 5.0 \\
\text { on) }\end{array}$ & Yes & Red, puffy face (duration unclear) \\
\hline $2 \mathrm{JIA}$ & $18(2.4)$ & $\begin{array}{l}\text { Intermittent high/burst ( } 4.3 \\
\text { on) }\end{array}$ & Yes & $\begin{array}{l}9+\text { months of weight gain, cushingoid facies, buffalo hump, } \\
\text { protruding abdomen with striae, diabetes, adrenal insufficiency, } \\
\text { and probable spinal compression fractures. }\end{array}$ \\
\hline $3 \mathrm{JIA}$ & $2(12.4)$ and 3 (17.9) & No & Yes & None \\
\hline 4 CSLE & No & Bursts $(0$, never halted) & Yes & None \\
\hline $5 \mathrm{JlA}$ & $1(1.1)$ & No & Yes & None \\
\hline $6 \mathrm{JIA}$ & $1(18.3)$ & Systemic (15.0) & Yes & $<1$ day of sweating, chills, and flushing (allergic reaction*) \\
\hline $7 \mathrm{JIA}$ & $2(9.2)$ & No & Yes & $2.5+$ months of cushingoid facies \\
\hline $8 \mathrm{MCTD}$ & $3(7.4)$ & $\begin{array}{l}\text { Maintenance }(0, \text { never } \\
\text { halted) }\end{array}$ & Yes & None \\
\hline $9 \mathrm{JA}$ & $7(0.8)$ & No & Yes & $\begin{array}{l}\text { Around } 9 \text { months of weight gain, increased appetite, moon facies, } \\
\text { and protruding abdomen }\end{array}$ \\
\hline Summary: & $\begin{array}{l}89 \% \text { received IASI } \\
8.3(0.8-18.3)\end{array}$ & $\begin{array}{l}56 \% \text { received systemic } \\
\text { steroids } 4.3(0-15.0)\end{array}$ & Yes & $44 \%$ experienced non-allergic adverse reactions to steroids* \\
\hline
\end{tabular}

*P6 has known steroid allergy

Key: $\mathrm{Pt}=$ Patient identifier and rheumatic disease, $\mathrm{IASI}=$ Intra-articular steroid injection

1 also continuing their prior $\mathrm{HCQ}) ; 1 \mathrm{ABA}, \mathrm{MTX}$, and ustekinumab; $1 \mathrm{ABA}$ and $\mathrm{HCQ} ; 1$ MTX and leflunomide; and $1 \mathrm{HCQ}$, MTX, and tocilizumab. One patient did not begin biologics nor DMARDs and another continued taking their prior HCQ. Collectively, six patients began the non-TNFai ABA (5) or tocilizumab (1) a median of 17.4 months (4.8-25.5) FHD, with 4 (3 ABA; 1 tocilizumab) patients having already resolved their histoplasmosis. One patient who was receiving ABA was transitioned to ustekinumab 33.6 months following $\mathrm{HC}$ (46.3 months FHD). Among the 2 patients still showing low, asymptomatic antigenemia at ABA initiation, 1 cleared their antigenemia after 37 months (16.6 after beginning $\mathrm{ABA}$ ) and the other patient remained antigen positive at $\geq 18$ months $\mathrm{FHD}$, although still asymptomatic at 25.4 months FHD. Additionally, 6/9 (67\%) patients initiated DMARDs. MTX was started by 3 patients a median of 22 months (11.2-51.2) FHD, with 1 patient still asymptomatically antigen-positive (11 months FHD) and 2 patients already cleared (median 20 months FHD). This positive patient cleared their antigenemia 26 months after beginning MTX. HCQ was initiated in 2 antigen-positive, asymptomatic patients at 6 and 10 months FHD and prior HCQ was continued in 2 others. These 2 positive patients had resolution of their antigenemia 15 and 27 months after beginning HCQ, respectively. Leflunomide was started in one patient taking MTX 25 months after HC (62 months FHD). The median time FDH to first taking DMARDs was 3.1 months (0 [still positive]-51.2) and to first beginning biologics was 17.4 months (4.8-25.5), while the median time following HC to DMARD and biologic initiation was 0 (0 to 9.4 ) and 2 months $(0-11)$, respectively (Table 4$)$.

\section{Discussion}

In this case series, we describe treatment and disease course of cRD diagnosed with histoplasmosis. Outcomes were favorable, with non-TNFai biologic and DMARD medications, including ABA and $\mathrm{HCQ}$, being safely reinitiated in patients requiring rheumatic disease alleviation. Although TNFai therapy complicated by granulomatous infections like histoplasmosis has been well-recognized [7, 17-20, 23], few published reports address reinstitution of IM treatments following histoplasmosis diagnosis $[8,19,22,23]$. Moreover, there is a lack of guidance on underlying disease management and long-term followup $[19,22]$. In this series, rheumatic diseases were initially controlled by NSAIDs and HCQ, but all cRD eventually needed additional treatments. Moreover, we observed that Cushingoid symptoms developed in almost half (4/9) of patients receiving corticosteroids during antifungal treatment. Thus, managing rheumatic disease during recovery from histoplasmosis poses challenges as IM are halted upon serious infections.

\section{Itraconazole and Steroids}

The often-chronic nature of fungal and rheumatic therapies make selecting compatible medications critical. In severe $\mathrm{DH}$, initial therapy may begin with intravenous 
Table 4 Reintroduction of immunosuppressive medications and patient histoplasmosis course. Patient history was examined for timing of systemic immunosuppressive medication reinitiation (localized steroid use was not included). Timing of reinitiation was compared with state of histoplasmosis, with "still +" denoting patient had not cleared their histoplasmosis infection based on antigenemia and/or clinical symptoms and "HC for \#" indicating how many months it had been since histoplasmosis clearance to beginning the immunosuppressive medication. Median months of follow-up since histoplasmosis clearance was 45.6 (14.7-78.9)

\begin{tabular}{|c|c|c|c|c|}
\hline $\mathrm{Pt}$ & \multicolumn{4}{|c|}{$\begin{array}{l}\text { Immunosuppressive Medication Resumption (months following histoplasmosis diagnosis, histoplasmosis status) } \\
\text { Months to HC }\end{array}$} \\
\hline $1 \mathrm{JA}$ & \multicolumn{4}{|c|}{$\begin{array}{l}\text { HCQ (10.0, still +), ABA (20.1, still +), Intermittent systemic steroid bursts ( } 5.0 \text { on, still +) } \\
\text { Time to HC: } 36.7\end{array}$} \\
\hline $2 \mathrm{JIA}$ & \multicolumn{4}{|c|}{$\begin{array}{l}\text { MTX (11.2, still +), Leflunomide (61.8, HC for 24.6), Stress/Intermittent steroid bursts }(4.3 \text {, still }+) \\
\text { Time to HC: } 37.2\end{array}$} \\
\hline $3 \mathrm{JA}$ & \multicolumn{4}{|c|}{$\begin{array}{l}\text { MTX (22.1, HC for 9.4), ABA (14.2, HC for 1.4), Ustekinumab (46.3, HC for 33.6) } \\
\text { Time to HC: } 12.7\end{array}$} \\
\hline 4 CSLE & \multicolumn{4}{|c|}{$\begin{array}{l}\text { HCQ }(0, \text { still }+) \text {, ABA (14.7, HC for 11.0), Low dose maintenance steroids (0, still }+ \text { ) } \\
\text { Time to HC: } 3.7\end{array}$} \\
\hline $5 \mathrm{JA}$ & \multicolumn{4}{|l|}{$\begin{array}{l}\text { ABA (4.8, HC for } 2.5) \\
\text { Time to HC: } 2.3\end{array}$} \\
\hline $6 \mathrm{JlA}$ & \multicolumn{4}{|c|}{$\begin{array}{l}\text { HCQ (6.2, still +), MTX (51.2, HC for 30.0), Tocilizumab (21.8, HC for 4.9), Systemic steroids (15.0, HC for 0.6) } \\
\text { Time to HC: } 21.2\end{array}$} \\
\hline $7 \mathrm{JA}$ & \multicolumn{4}{|l|}{$\begin{array}{l}\text { ABA }(25.5 \text {, still }+) \\
\text { Antigen positive }<\text { LOQ at } 18+\end{array}$} \\
\hline 8 MCTD & \multicolumn{4}{|l|}{$\begin{array}{l}\text { HCQ }(0, \text { still }+) \text {, Low dose stress steroids }(0, \text { still }+) \\
\text { Antigen positive and decreasing at } 11+\end{array}$} \\
\hline $9 \mathrm{JA}$ & \multicolumn{4}{|l|}{$\begin{array}{l}\text { None } \\
\text { Time to HC: } 3.3\end{array}$} \\
\hline Summary: & $\begin{array}{l}\text { Total patients resuming IM: 8/9 (89), with 6/9 (67\%) DMARD; } \\
6 / 9(67 \%) \text { biologics; and 5/9 (56\%) systemic steroids }\end{array}$ & $\begin{array}{l}\text { Collective median } \\
\text { (range) after diagnosis: } \\
\text { DMARDs } 3.1 \text { (0-51.2) } \\
\text { Biologic 17.4 (4.8-25.5) } \\
\text { Corticosteroid } 4.3 \text { (0- } \\
\text { 15.0) }\end{array}$ & $\begin{array}{l}\text { Collective median } \\
\text { (range) to IM use from } \\
\text { HC: } \\
\text { DMARD Still + (Still + to } \\
9.4 \text { ) } \\
\text { Biologic } 2 \text { (Still + to 11.0) } \\
\text { Corticosteroid all still + }\end{array}$ & $\begin{array}{l}\text { Pt median (range) to } \\
\text { HC: } \\
12.7(2.3-37.2) \text {, with } 2 \\
\text { still low positives }\end{array}$ \\
\hline
\end{tabular}

Key: $\mathrm{Pt}=$ Patient identifier and rheumatic disease; $\mathrm{IM}=$ Immunosuppressive medication; $\mathrm{HCQ}=$ hydroxychloroquine; $\mathrm{ABA}=\mathrm{abatacept} ; \mathrm{MTX}=\mathrm{methotrexate}, \mathrm{HC}=$ histoplasmosis clearance; LOQ = Limit of quantification; + = positive

amphotericin transitioning to oral itraconazole, with a total antifungal duration of at least 12 months [7, 21]. Itraconazole is an effective antifungal, but it potently inhibits the CYP3A4 pathway, thereby decreasing steroid metabolism $[8,9,11,12]$. Nevertheless, adjunctive corticosteroids may still be indicated for fungal infections and other inflammatory states, like rheumatic flares [1, $7,8]$. Furthermore, systemic or local corticosteroid use by itself can also cause iatrogenic Cushing's syndrome, although drug interactions increase risk [9, 28, 29]. Shortly following IASIs, $44 \%$ of our cRD developed nonallergic adverse reactions with prominent Cushingoid features. To our knowledge, this is the first description of Cushing's syndrome in cRD undergoing itraconazole treatment following IASI and systemic steroids. However, previous reports associating itraconazole and steroid therapy with hypothalamic-pituitary-adrenal (HPA) axis suppression and, more infrequently, overt Cushing's syndrome have been published, usually for patients with cystic fibrosis taking inhaled steroids [9, 10, 13, 15].

The somewhat surprisingly high fraction of patients presenting with overt Cushingoid features in our report could perhaps be due to differences in systemic steroid levels, route of steroid administration, type of steroid, underlying disease, and/or drug interactions. For example, a study comparing the effects of itraconazole on a single dose of methylprednisolone versus prednisone in healthy volunteers indicated prednisone's pharmacokinetics were unaffected while methylprednisolone concentration increased [12]. In our limited cohort, is difficult to draw firm conclusions about what/if combinations of corticosteroids and itraconazole place patients at greatest risk for HPA axis-related syndromes. High dose steroidal bursts combined with IASI perhaps contribute, but more research is needed, for example, to determine if route of steroid administration matters and if underlying comorbidities affect risk. Regardless, there is an urgency to determine optimal strategies of IM usage in the setting of concurrent histoplasmosis that facilitate histoplasmosis recovery and rheumatic disease management. Moreover, perhaps HPA axis monitoring should be considered in patients needing itraconazole and steroids [13]. 


\section{Histoplasmosis and Immunosuppressants}

Acute DH develops in about 1 of every 2000 acute histoplasmosis cases, and most acute DH occurs in immunosuppressed patients [30]. Markedly, immunocompromised patients (e.g., AIDS) have a 10-fold increased risk for developing $\mathrm{DH}$ compared to immunocompetent persons, and DH mortality rates in severely immunocompromised patients can reach $30 \%$ [31-33]. Chu et al. noted that in 111 pediatric cases of histoplasmosis requiring hospitalization, $32 \%$ of these patients were immunocompromised and/or had a comorbidity with an overall pediatric mortality of $5 \%$ [33]. Likewise, a review of 73 pediatric histoplasmosis cases by Ouellette et al. reported immunocompromised children had a $2.5 \mathrm{X}$ higher rate (56\% vs. $21 \%$ ) of developing DH [34]. In line with these findings, in our series most (66\%) $\mathrm{PH}$ cases were in patients without prior IM, while all patients with DH were receiving prior combination or DMARD therapy.

Studies assessing post-histoplasmosis care in patients experiencing TNFai complicated by histoplasmosis are limited [19, 20, 22, 23]. Notably, Vergidis et al. described outcomes of 98 patients with various autoimmune diseases diagnosed with histoplasmosis, wherein 3/98 patients continued non-combination TNFai, 11/58 patients continued DMARDs, and 13/23 patients continued corticosteroids. The 3 histoplasmosis cases maintaining TNFai, which is not the standard of care, were described as "mild" and all patients achieved remission after 6-10 months of itraconazole. During follow-up (median 32 months), 2/25 patients resuming TNFai (and 1/49 who had not) developed histoplasmosis recurrence with 1 fatality (taking TNFai). Follow-up data were unavailable for the other patients, and resumption information on DMARDs and corticosteroids was not reported [22]. Furthermore, after TNFai was held in 14/15 adult patients with RA, 4 resumed them at least 5 months FHD. The patient continuing TNFai, whose lung biopsy showed inactive disease, did well, while one patient who resumed TNFai developed clinical recurrence, permanently halted it, and recovered [23]. In our case series, persistent rheumatic disease activity led to resumption of biologics and non-HCQ DMARDs in 1 cSLE and 6 JIA patients FHD. However, no patients were restarted on TNFai due to safety concerns even if histoplasmosis disease was mild. One JIA patient did not require IM and the patient with MCTD proceeded on their pre-histoplasmosis regimen of HCQ and maintenance steroids. The 3 patients resuming MTX had favorable outcomes. HCQ was continued without issue for the CSLE and MCTD patients, and HCQ was an alternative (or additive) treatment option for JIA patients. Unlike some evidence on MTX, HCQ does not increase infection risk, but all 3 patients starting either medicine cleared their histoplasmosis without recurrence.
Considering timing of biologic reinitiation FHD, 5 cRD began the non-TNFai ABA before [2] or following [3] $\mathrm{HC}$, while the initiations of tocilizumab or ustekinumab were only after $\mathrm{HC}$. Although no patients were receiving $\mathrm{ABA}$, tocilizumab, or ustekinumab prior to their histoplasmosis diagnosis, all $4 \mathrm{cRD}$ beginning them have remained free of histoplasmosis recurrence and neither patient starting ABA while still antigen positive experienced worsening of their histoplasmosis. Moreover, 1 of these $2 \mathrm{ABA}$ patients has achieved $\mathrm{HC}$ to date. Despite the small numbers, the use of $A B A$ in these cRD resulted in good rheumatologic outcomes without exacerbating the infection, suggesting that ABA may be an option for treating rheumatic patients with histoplasmosis. The inability to support some cRD without higher tier medications underscores the need to promote infection safety while controlling rheumatic disease. For example, considering lower risk (non-TNFai) biologics, like $\mathrm{ABA}$, for patients in endemic fungal areas such as the Ohio and Mississippi River Valleys where histoplasmosis is the most frequent TNFai-associated endemic fungal infection [6]. While screening for histoplasmosis prior to beginning biologics is not routinely recommended, nevertheless physicians prescribing IM must be aware of histoplasmosis presentations and risk factors [1].

\section{Conclusions}

Histoplasmosis is an important complication of the immunosuppressive therapy utilized to treat cRD in endemic areas. This small case series proposes corticosteroids, non-HCQ DMARDs, and non-TNFai biologic treatments initiated months FHD or after HC did not appear to induce histoplasmosis recurrence, and, for a majority of patients, permitted histoplasmosis antigen clearance. While receiving antifungal therapy, necessary intra-articular or systemic steroid usage may potentially merit HPA axis monitoring. However, firm conclusions from our report are limited due to the small number of patients and varying IM therapies. Given the crucial role of IMs like biologics and DMARDs in cRD care, additional studies are needed to examine the long-term safety and efficacy of ABA, tocilizumab, ustekinumab, MTX, HCQ, and corticosteroids during histoplasmosis treatment and recovery.

\footnotetext{
Abbreviations

JIA: Juvenile idiopathic arthritis; TNFai: Tumor necrosis factor alpha inhibitor; IL-1b: Interleukin-1 beta; IL-1R: Interleukin-1 receptor; IL-6R: Interleukin-6 receptor; ABA: Abatacept; IM: Immunosuppressive medications; $H$. capsulatum: Histoplasmosis capsulatum; CRD: Children with rheumatic disease; NSAIDs: Nonsteroidal anti-inflammatory drugs; DMARDs: Diseasemodifying anti-rheumatic drugs; MTX: Methotrexate; HCQ: Hydroxychloroquine; RA: Rheumatoid arthritis; FHD: Following histoplasmosis diagnosis; HC: Histoplasmosis clearance; NCH: Nationwide Children's Hospital; IASIs: Intra-articular steroid injections; ERA: Enthesitisrelated arthritis; CSLE: Childhood-onset systemic lupus erythematosus;
} 
MCTD: Mixed connective tissue disease; DH: Disseminated histoplasmosis; PH: Pulmonary histoplasmosis; HPA: Hypothalamic-pituitary-adrenal

\section{Acknowledgements}

We thank our patients and families at $\mathrm{NCH}$ for their courage and inspiration and our pediatric rheumatology colleagues for their valuable input.

\section{Authors' contributions}

RB wrote the first draft of the manuscript. RB and SA contributed equally to this study. Each author listed on the manuscript has seen and approved the submission of this version of the manuscript and takes full responsibility for the manuscript. The author(s) read and approved the final manuscript.

\section{Funding}

No honorarium, grant, or other form of payment was given to anyone to produce the manuscript.

\section{Availability of data and materials}

The datasets analyzed during the current study available from the corresponding author on reasonable request.

\section{Declarations}

\section{Ethics approval and consent to participate}

An Institutional Review Board was obtained from Nationwide Children's Hospital IRB17-01241.

\section{Consent for publication}

Not applicable.

\section{Competing interests}

There are no conflicts of interest.

\section{Author details}

${ }^{1}$ The Ohio State University College of Medicine, OH, Columbus, USA. ${ }^{2}$ Nationwide Children's Hospital, Ohio, Columbus, USA. ${ }^{3}$ Department of Pediatric Rheumatology, Nationwide Children's Hospital, 555 S. 18th St, OH 43205 Columbus, USA

Received: 28 January 2021 Accepted: 25 May 2021

\section{Published online: 07 June 2021}

\section{References}

1. Higgins GC. Complications of Treatments for Pediatric Rheumatic Diseases. Pediatr Clin North Am. 2018;65(4):827-54.

2. Lee $\mathrm{YH}$, et al. Comparative efficacy and safety of tocilizumab, rituximab, abatacept and tofacitinib in patients with active rheumatoid arthritis that inadequately responds to tumor necrosis factor inhibitors: a Bayesian network meta-analysis of randomized controlled trials. Int J Rheum Dis. 2016:19(11):1103-11.

3. Gerriets $V$, et al. Tumor Necrosis Factor Inhibitors. StatPearls [Internet]. Treasure Island (FL): StatPearls Publishing; [Updated 2020 Jul 4].

4. Manos NE, et al. Geographic variation in the prevalence of histoplasmin sensitivity. Dis Chest. 1956;29(6):649-68.

5. Zumla A, et al. Granulomatous infections: etiology and classification. Clin Infect Dis. 1996;23(1):146-58.

6. Smith JA, et al. Endemic Fungal Infections in Patients Receiving Tumour Necrosis Factor-a Inhibitor Therapy. Drugs. 2009;69(11):1403-15.

7. Hage CA, et al. Histoplasmosis: Up-to-Date Evidence-Based Approach to Diagnosis and Management. Semin Respir Crit Care Med. 2015;36(5):729-45.

8. Wheat $L$, et al. Clinical practice guidelines for the management of patients with histoplasmosis: 2007 update by the Infectious Diseases Society of America. Clin Infect Dis. 2007:45(7):807-25.

9. De Wachter $E$, et al. Inhaled budesonide induced Cushing's syndrome in cystic fibrosis patients, due to drug inhibition of cytochrome P450. J Cyst Fibros. 2003:2(2):72-5.

10. Parmar JS, et al. Profound adrenal suppression secondary to treatment with low dose inhaled steroids and itraconazole in allergic bronchopulmonary aspergillosis in cystic fibrosis. Thorax. 2002;57(8):749-50.
11. Varis T, et al. Itraconazole decreases the clearance and enhances the effects of intravenously administered methylprednisolone in healthy volunteers. Pharmacol Toxicol. 1999;85(1):29-32.

12. Lebrun-Vignes $B$, et al. Effect of itraconazole on the pharmacokinetics of prednisolone and methylprednisolone and cortisol secretion in healthy subjects. Br J Clin Pharmacol. 2001;51(5):443-50.

13. Skov $M$, et al. latrogenic adrenal insufficiency as a side-effect of combined treatment of itraconazole and budesonide. Eur Respir J. 2002;20(1):127-33.

14. Philips MR. Methotrexate and Ras methylation: a new trick for an old drug? Sci STKE. 2004;2004(225):pe13.

15. Gilchrist FJ, et al. Itraconazole and inhaled fluticasone causing hypothalamic-pituitary-adrenal axis suppression in adults with cystic fibrosis. J Cyst Fibros. 2013;12(4):399-402.

16. Solomon $\mathrm{DH}$, et al. Adverse Effects of Low-Dose Methotrexate: $\mathrm{A}$ Randomized Trial. Ann Intern Med. 2020;172(6):369-80.

17. Tsiodras $S$, et al. Fungal infections complicating tumor necrosis factor alpha blockade therapy. Mayo Clin Proc. 2008;83(2):181 - 94.

18. Park $\mathrm{S}$, et al. Cholestasis and disseminated histoplasmosis in a psoriatic patient on infliximab: case report and review of literature. BMC Gastroenterol. 2020:20(1):141.

19. Dotson $\mathrm{J}$, et al. Presentation and outcome of histoplasmosis in pediatric inflammatory bowel disease patients treated with antitumor necrosis factor alpha therapy: a case series. Inflamm Bowel Dis. 2011;17(1):56-61.

20. Hage CA, et al. Recognition, diagnosis, and treatment of histoplasmosis complicating tumor necrosis factor blocker therapy. Clin Infect Dis. 2010;50:85-92.

21. Schwartz IS, et al. Endemic Mycoses: What's New About Old Diseases? Curr Clin Microbiol Rep. 2016;3(2):71-80

22. Vergidis $\mathbf{P}$, et al. Histoplasmosis complicating tumor necrosis factor-a blocker therapy: a retrospective analysis of 98 cases. Clin Infect Dis. 2015 Aug 1;61(3):409 - 17

23. Olson TC, et al. Histoplasmosis infection in patients with rheumatoid arthritis, 1998-2009. BMC Infect Dis. 2011;11:145

24. Baddley JW, et al. ESCMID Study Group for Infections in Compromised Hosts (ESGICH) Consensus Document on the safety of targeted and biological therapies: an infectious diseases perspective (Soluble immune effector molecules [1]: anti-tumor necrosis factor-alpha agents). Clin Microbiol Infect. 2018;24(Suppl 2):10-20

25. Winthrop KL, et al. ESCMID Study Group for Infections in Compromised Hosts (ESGICH) Consensus Document on the safety of targeted and biological therapies: an infectious diseases perspective (Soluble immune effector molecules [II]: agents targeting interleukins, immunoglobulins and complement factors). Clin Microbiol Infect. 2018;24(Suppl 2):21-40.

26. Ozen $\mathrm{G}$, et al. Safety of abatacept compared with other biologic and conventional synthetic disease-modifying antirheumatic drugs in patients with rheumatoid arthritis: data from an observational study. Arthritis Res Ther. 2019:21(1):141.

27. Donnelly JP, et al. Revision and Update of the Consensus Definitions of Invasive Fungal Disease From the European Organization for Research and Treatment of Cancer and the Mycoses Study Group Education and Research Consortium. Clin Infect Dis. 2020;71(6):1367-76.

28. Sukhumthammarat W, et al. Local Injection of Triamcinolone Acetonide: A Forgotten Aetiology of Cushing's Syndrome. J Clin Diagn Res. 2017;11(6):OR01-2

29. Alkhuder L, et al. Infantile latrogenic Cushing Syndrome due to Topical Steroids. Case Reports in Pediatrics, vol. 2019, 4 pages. Article ID 2652961.

30. Pietrobon D, et al. Disseminated histoplasmosis and AIDS in an Argentine hospital: clinical manifestations, diagnosis and treatment. Enferm Infecc Microbiol Clin. 2004:22(3):156-9.

31. Wheat $L$, et al. Cavitary histoplasmosis occurring during two large urban outbreaks. Analysis of clinical, epidemiologic, roentgenographic, and laboratory features. Medicine. 1984;63(4):201-9.

32. Colombo AL, et al. Epidemiology of endemic systemic fungal infections in Latin America. Med Mycol. 2011:49(8):785-98.

33. Chu JH, et al. Hospitalizations for endemic mycoses: a population-based national study. Clin Infect Dis. 2006:42(6):822-5.

34. Ouellette CP, et al. Pediatric Histoplasmosis in an Area of Endemicity: A Contemporary Analysis. J Pediatric Infect Dis Soc. 2019:8(5):400-7.

\section{Publisher's Note}

Springer Nature remains neutral with regard to jurisdictional claims in published maps and institutional affiliations. 